Background Currently, most neoantigen pipelines often focus on the detection of neoantigens derived from mutations in the coding regions of the genome. However, in some cancer indications, the number of mutations detectable in tumours can be very low (low tumour mutational burden). This limits the number of actionable neoantigens and results in so-called 'cold' tumours. In these cases, non-canonical neoantigens resulting from alterations in non-coding regions of the human genome could represent a high potential alternative for treatment.

Indeed, recent research has revealed that previously presumed non-coding regions of the human genome, such as long non-coding RNAs (lncRNAs), can contain translatable small open reading frames (smORFs) generating micropeptides. Some of these micropeptides have already been shown to be involved in cancer development, but these small peptides could also represent a high potential source of non-canonical neoantigens for personalised therapy.

Materials and Methods Here, we present smORFin, a machine learning algorithm specifically trained to identify smORFs in transcripts and to assess their coding potential. While most tools are focused on longer sequences, smORFin is specifically developed to target small ORFs $(<303$ nucleotides). Furthermore, smORFin also accounts for smORFs with alternative initiation codons, thereby improving its sensitivity for the detection of novel unannotated smORFs.

In addition, the impact of mutations in allegedly non-coding regions of tumour genomes and its influence on the neoantigen repertoire, was evaluated through integration of smORFin in a neoantigen identification pipeline targeting lncRNA-derived mutated epitopes; lncRNeos.

Results The smORFin model reaches a precision of 0.98 and an accuracy of 0.95 on its testing dataset. Using this new prediction tool, a library of human smORFs was assembled, the so-called smORFeome. This library of smORFs, and their associated proteins, was evaluated as a reference for spectrum to peptide matching in mass spectrometry data (MS) analysis. Indeed, the evaluation of seven MS samples revealed and validated the presence of smORFeome-related micropeptides and HLA-I associated epitopes originating from smORFs.

Furthermore, it was observed that IncRNA-derived epitopes only represent a minor fraction of the total neoantigen load. Strikingly, when only focusing on tumours with a low neoantigen load, IncRNeos represented up to $27 \%$ of the total neoantigen load. This indicates that for tumours with a low TMB, and therefore with a low neoantigen load, lncRNeos allows to significantly expand the neoantigen repertoire. Biological in vivo/in vitro validation remains necessary to assess the existence, presentation, and actionability of lncRNeos.

Conclusions A novel random forest-based algorithm was developed to address the need for reliable identification of lncRNA-born smORFs. Furthermore, the integration of this prediction algorithm in a neoantigen pipeline allowed the identification of lncRNA-derived neoantigens and marks them as a potential novel source for personalised immunotherapy.

Disclosure Information C. Bogaert: None. L. Van Oudenhove: None. B. Fant: None.

\section{P03.06 DECIPHERING VACCINE-INDUCED NEOEPITOPE-SPECIFIC T CELL RESPONSES IN A PATIENT WITH H3K27M- MUTANT MIDLINE GLIOMA WHO GRADE 4}

1,2 K Lindner* ${ }^{3} \mathrm{~K}$ Kromer, ${ }^{1} \mathrm{~K}$ Jähne 1,4 ${ }^{3} \mathrm{JM}$ Lindner, ${ }^{1,2,4} \mathrm{M}$ Platten, ${ }^{1,4} \mathrm{~K}$ Sahm. ${ }^{1} \mathrm{DKFZ}$ Heidelberg, Heidelberg, Germany; ${ }^{2}$ National Center for Tumor Diseases (NCT), Heidelberg, Germany; ${ }^{3}$ BioMed X, Heidelberg, Germany; ${ }^{4}$ Department of Neurology, Medical Faculty Mannheim, Mannheim, Germany

\subsection{6/jitc-2021-ITOC8.28}

Background K27M-mutant histone-3 (H3K27M) defines a clinically and molecularly distinct entity of diffuse midline gliomas WHO grade 4 with an unfavorable prognosis. From an immunological perspective, H3K27M constitutes a cancer neoepitope: in a syngeneic MHC-humanized mouse model, an H3K27M-specific long peptide vaccine induced mutation-specific $\mathrm{T}$ cells responses capable of inhibiting growth of H3K27M-expressing tumors.

Materials and Methods Here, we describe clinical response of a patient diagnosed with H3K27M-mutant midline gliomas to H3K27M-specific peptide vaccination and exploit vaccineinduced $\mathrm{T}$ cell phenotypes.

Results Repeated peptide vaccinations were well tolerated and resulted in long-term response after pseudoprogression. Longitudinal immune monitoring showed induction of $\mathrm{H} 3 \mathrm{~K} 27 \mathrm{M}$ specific CD4-driven $\mathrm{T}$ cell responses in the peripheral blood. Within the cerebrospinal fluid, expansion of HLA-specific vaccine-induced $\mathrm{T}$ cell receptor (TCR) clones was observed and associated with distinct HLA types.

Conclusions Identification of vaccine-induced TCR clones within the peripheral blood and CSF of patients with H3K27M-mutant midline glioma may be used to prioritize TCRs for adoptive $\mathrm{T}$ cell therapy.

Disclosure Information K. Lindner: None. K. Kromer: None. K. Jähne: None. L. Bunse: E. Ownership Interest (stock, stock options, patent or other intellectual property); Modest; Patent: US20180155403A1 (Histone Anti-Cancer Vaccines). C. Tan: None. I. Poschke: None. E. Green: None. J.M. Lindner: None. M. Platten: C. Other Research Support (supplies, equipment, receipt of drugs or other in-kind support); Modest; Roche. E. Ownership Interest (stock, stock options, patent or other intellectual property); Modest; Patent: US20180155403A1 (Histone Anti-Cancer Vaccines). K. Sahm: E. Ownership Interest (stock, stock options, patent or other intellectual property); Modest; Patent: US20180155403A1 (Histone Anti-Cancer Vaccines).

\section{P03.07 ANALYSIS OF SCRNASEQ FROM THE HUMAN THYMUS NOMINATES GENES POTENTIALLY MISSING FROM CENTRAL TOLERANCE OF CYTOTOXIC T CELLS}

L Blumenberg*, G Atwal, A Dhanik. Regeneron Pharmaceuticals, Tarrytown, NY, USA

\subsection{6/jitc-2021-ITOC8.29}

Background During thymic development, cytotoxic T cells that can bind to and attack self antigens undergo negative selection thus preventing damage to the self tissues. The sparse medullar thymic epithelial cells (mTECs) present in the thymus are responsible for presenting self antigens to $\mathrm{T}$ cells so that they 